\title{
OPTIMAL STOPPING RULE FOR THE NO-INFORMATION DURATION PROBLEM WITH RANDOM HORIZON
}

\author{
MITSUSHI TAMAKI,* Aichi University
}

\begin{abstract}
As a version of the secretary problem, Ferguson, Hardwick and Tamaki (1992) considered an optimal stopping problem called the duration problem. The basic duration problem is the classical duration problem, in which the objective is to maximize the time of possession of a relatively best object when a known number of rankable objects appear in random order. In this paper we generalize this classical problem in two directions by allowing the number $N$ (of available objects) to be a random variable with a known upper bound $n$ and also allowing the objects to appear in accordance with Bernoulli trials. Two models can be considered for our random horizon duration problem according to whether the planning horizon is $N$ or $n$. Since the form of the optimal rule is in general complicated, our main concern is to give to each model a sufficient condition for the optimal rule to be simple. For $N$ having a uniform, generalized uniform, or curtailed geometric distribution, the optimal rule is shown to be simple in the so-called secretary case. The asymptotic results, as $n \rightarrow \infty$, will also be given for these priors.
\end{abstract}

Keywords: Secretary problem; best-choice problem; Bruss' odds theorem; simple rule; candidate; relative rank; Bernoulli trial

2010 Mathematics Subject Classification: Primary 60G40; 62L15

\section{Introduction}

In the classical best-choice problem, a version of the secretary problem studied extensively in Gilbert and Mosteller (1966), a fixed known number $n$ of rankable objects ( 1 being the best and $n$ the worst) appear one at a time in random order with all $n$ ! permutations equally likely. Each time an object appears, we observe only the relative rank of the current object with respect to its predecessors. We must select one object and find a stopping rule that maximizes the probability of selecting the best object. The optimal rule passes over the first $s_{c}^{*}(n)-1$ objects and stops with the first relatively best object if any, where

$$
s_{c}^{*}(n)=\min \left\{k \geq 1: \sum_{j=k+1}^{n} \frac{1}{j-1} \leq 1\right\} .
$$

As $n \rightarrow \infty, s_{c}^{*}(n) / n \rightarrow \mathrm{e}^{-1} \approx 0.3679$ and the optimal probability of selecting the best overall also converges to $\mathrm{e}^{-1}$.

As a different version of the secretary problem, Ferguson et al. (1992) considered an optimal stopping problem called the duration problem. Among other models, the basic duration problem is the classical duration problem (see Section 2.2 of Ferguson et al. (1992), in which this

Received 3 February 2012; revision received 12 January 2013.

* Postal address: Department of Business Administration, Aichi University, Nagoya Campus, Hiraike 4-60-6, Nakamura, Nagoya, Aichi 453-8777, Japan. Email address: tamaki@ vega.aichi-u.ac.jp 
problem is called the finite-horizon duration problem), where we must find a stopping rule that maximizes the expected duration of holding a relatively best object. Clearly, we select only a relatively best object, receiving a payoff of 1 plus the number of future observations before a new relatively best object appears or until the final stage $n$ is reached. The optimal rule passes over the first $t_{c}^{*}(n)-1$ objects and stops with the first relatively best object if any, where

$$
t_{c}^{*}(n)=\min \left\{k \geq 1: \sum_{j=k+2}^{n} \frac{1}{j-1} \sum_{i=j}^{n} \frac{1}{i} \leq \sum_{i=k+1}^{n} \frac{1}{i}\right\} .
$$

As $n \rightarrow \infty, t_{c}^{*}(n) / n \rightarrow \mathrm{e}^{-2} \approx 0.1353$ and the optimal proportional payoff (equal to the payoff $/ n$ ) converges to $2 \mathrm{e}^{-2} \approx 0.2707$.

The optimal stopping rule in each of the above two classical problems is simple in the sense that it stops on the first relatively best object appearing after a given stage. Henceforth, such a rule is referred to as a simple rule. We call $s_{c}^{*}(n)$ and $t_{c}^{*}(n)$ threshold values.

In this paper we attempt to generalize the classical duration problem in two directions by allowing the number of objects to be random and also allowing the objects to appear in accordance with Bernoulli trials. More specifically, this can be stated as follows, if we call an object candidate when it is relatively best. Each object observed is immediately judged either to be a candidate or not. Let $X_{j}, j \geq 1$, be the indicator of the event that the $j$ th object is a candidate, and suppose that $X_{1}, X_{2}, \ldots$ is a sequence of independent Bernoulli random variables with $\mathbb{P}\left\{X_{j}=1\right\}=a_{j}=1-\mathbb{P}\left\{X_{j}=0\right\}, j \geq 1$. Also, let $N$ be a bounded random variable representing the number of available objects, i.e. the length of the random horizon. It is assumed that $N$ is independent of the sequence $X_{1}, X_{2}, \ldots$ and has a prior distribution $p_{k}=\mathbb{P}\{N=k\}$ such that $\sum_{k=1}^{n} p_{k}=1$ and $p_{n}>0$ for a known upper bound $n$, and that the payoff is 0 if no object is chosen. This problem, henceforth referred to as the random-horizon duration problem (RHDP), is completely specified by two sequences $\left\{a_{j}\right\}_{j=1}^{n}$ and $\left\{p_{k}\right\}_{k=1}^{n}$, and involves finding a stopping rule that maximizes the expected duration of holding a candidate based on the available information $X_{1}, X_{2}, \ldots, X_{N}$.

The classical duration problem occurs as a special case of the RHDP if $N$ degenerates to $n$, i.e. $\mathbb{P}\{N=n\}=1$ and $a_{j}=1 / j, 1 \leq j \leq n$, because the relative ranks $R_{1}, R_{2}, \ldots, R_{n}$ of $n$ rankable objects presented one by one in random order have the property that the $R_{j}$ are independent with $\mathbb{P}\left\{R_{j}=i\right\}=1 / j, 1 \leq i \leq j$, and the only relevant information about $R_{j}$ is whether $R_{j}$ takes the value 1 or not. We are said to be in the secretary case if $a_{j}=1 / j, 1 \leq j \leq n$.

In Section 2 we formulate the RHDP. This problem can be distinguished as two models, model 1 and model 2, according to whether the final stage of the planning horizon is $N$ or $n$. This distinction is related to the last candidate. That is, it is assumed that if the chosen object is the last candidate prior to $N$, we can hold it until stage $N$ in model 1, and until stage $n$ in model 2 . It is easy to see that the optimal rule is always simple for $n \leq 3$. However, for $n \geq 4$, the form of the optimal rule heavily depends on $\left\{a_{j}\right\}_{j=1}^{n}$ and $\left\{p_{k}\right\}_{k=1}^{n}$, implying that the optimal rule is not necessarily simple (see Section 2 for an example where $N$ degenerates to $n=4$ ). Hence, our main concern in this paper is to give a sufficient condition for the optimal rule to be simple. Model 1 will be considered in Section 3 and model 2 in Section 4. An interesting application of this condition appears in the secretary case. For $N$ having a uniform, generalized uniform, or curtailed geometric distribution, the optimal rule is shown to be simple. The asymptotic results, as $n \rightarrow \infty$, will also be obtained for these prior distributions. The RHDP is related to the problem of choosing the last candidate considered in Bruss (2000). In Section 2, it is shown that we should stop earlier than in the corresponding Bruss problem. 
It is worth mentioning the similarity between the duration problem and the best-choice problem. Ferguson et al. (1992) recognized, in the secretary case, the similarity between the classical duration problem and the best-choice problem with a random number of objects having a uniform distribution on $\{1, \ldots, n\}$, first studied by Presman and Sonin (1972), and later studied by Samuels (2004), who gave a good explanation for this similarity. For such a similarity, see also Pearce et al. (2012) for multiple-choice problems, and Samuels (2004), Gnedin (2004), and Mazalov and Tamaki (2006) for a informed version of the problems. Gnedin (2005) extended this similarity to the equivalence between the probability maximization problem of stopping at the maximum in a random sequence and the associated duration problem of maximizing the interrecord time. In terms of our framework, Gnedin also showed that the RHDP with a given distribution of $N$ (as model 1) is equivalent to the probability maximization problem of choosing the last candidate but with some other distribution of the horizon (see Remark 3.1). Though Gnedin (2005) was the first to refer to the RHDP, he was mainly concerned with establishing and extending the equivalence relation and left untouched the problem of deriving the optimal rule of the RHDP.

\section{The formulation of the RHDP}

For the RHDP having the sequences $\left\{a_{j}\right\}_{j=1}^{n}$ and $\left\{p_{k}\right\}_{k=1}^{n}$, we write $b_{j}=1-a_{j}, 1 \leq j \leq n$, and introduce the notation

$$
B_{k, i}=b_{k+1} b_{k+2} \cdots b_{i}, \quad 0 \leq k<i \leq n,
$$

with $B_{k, k}=1$ for convenience, and

$$
\pi_{k}=p_{k}+p_{k+1}+\cdots+p_{n}, \quad 1 \leq k \leq n,
$$

with $\pi_{1}=1$ and $\pi_{n}>0$. Furthermore, define

$$
\sigma_{k}=\pi_{k}+(n-k) p_{k} .
$$

To avoid any unnecessary complication, we assume that $0<a_{j}<1$ for $j \geq 1$ throughout this paper except for the secretary case.

Denote by $k$ the state, where we have just observed the $k$ th object to be a candidate, $1 \leq$ $k \leq n$. Let $S(k)$ and $C(k)$ represent the expected payoff earned by stopping with the current candidate in state $k$ and by continuing observations in an optimal manner, respectively. Then $V(k)=\max \{S(k), C(k)\}$ represents the optimal expected payoff, provided that we start from state $k$. Defining $T_{k}$ as the time of the first candidate after $k$ if there is one, and as $N+1$ (as $n+1$ ) if there is none in model 1 (in model 2), we can formally express $S(k)$ as

$$
S(k)=\mathbb{E}\left[T_{k}-k \mid N \geq k\right] .
$$

The following lemma gives the explicit form of $S(k)$.

Lemma 2.1. We have, for $1 \leq k \leq n$,

$$
\begin{array}{ll}
S(k) & =\frac{1}{\pi_{k}} \sum_{i=k}^{n} \pi_{i} B_{k, i} \quad \text { for model } 1, \\
S(k) & =\frac{1}{\pi_{k}} \sum_{i=k}^{n} \sigma_{i} B_{k, i} \quad \text { for model } 2 .
\end{array}
$$


Proof. To clarify the dependence on the model, we write $S_{1}(k)$ or $S_{2}(k)$ for $S(k)$ corresponding to model 1 and model 2.

Model 1. Possession of the chosen candidate is terminated either by an arrival of a new candidate or by termination of the random horizon, whichever occurs first. Hence, $T_{k}$ takes the value $i$ on the event $E_{i} \cup F_{i}$, where $E_{i}$ and $F_{i}$ are mutually exclusive and defined as

$$
E_{i}=\left\{X_{k+1}=\cdots=X_{i-1}=0, X_{i}=1, \text { and } N \geq i\right\}
$$

and

$$
F_{i}=\left\{X_{k+1}=\cdots=X_{i-1}=0 \text { and } N=i-1\right\},
$$

respectively, for $k+1 \leq i \leq n+1$ with the interpretation that $E_{n+1}=\varnothing$. Thus, we have from (2.1),

$$
\begin{aligned}
S_{1}(k) & =\sum_{i=k+1}^{n+1}(i-k) \mathbb{P}\left\{T_{k}=i \mid N \geq k\right\} \\
& =\sum_{i=k+1}^{n}(i-k) \mathbb{P}\left\{E_{i} \mid N \geq k\right\}+\sum_{i=k+1}^{n+1}(i-k) \mathbb{P}\left\{F_{i} \mid N \geq k\right\} .
\end{aligned}
$$

Since $N$ and the $X_{j}$ are independent, we have

$$
\mathbb{P}\left\{E_{i} \mid N \geq k\right\}=B_{k, i-1} a_{i} \frac{\pi_{i}}{\pi_{k}}=\left(B_{k, i-1}-B_{k, i}\right) \frac{\pi_{i}}{\pi_{k}}
$$

and

$$
\mathbb{P}\left\{F_{i} \mid N \geq k\right\}=B_{k, i-1} \frac{p_{i-1}}{\pi_{k}}=B_{k, i-1} \frac{\pi_{i-1}-\pi_{i}}{\pi_{k}}
$$

with $\pi_{n+1}=0$. Substituting (2.5) and (2.6) into (2.4) yields

$$
\begin{aligned}
\pi_{k} S_{1}(k) & =\sum_{i=k+1}^{n}(i-k)\left(B_{k, i-1}-B_{k, i}\right) \pi_{i}+\sum_{i=k+1}^{n+1}(i-k) B_{k, i-1}\left(\pi_{i-1}-\pi_{i}\right) \\
& =\sum_{i=k}^{n} B_{k, i} \pi_{i},
\end{aligned}
$$

which proves (2.2).

Model 2. In contrast to (2.4), for this model, we can write

$$
S_{2}(k)=\sum_{i=k+1}^{n}(i-k) \mathbb{P}\left\{E_{i} \mid N \geq k\right\}+\sum_{i=k+1}^{n+1}(n+1-k) \mathbb{P}\left\{F_{i} \mid N \geq k\right\},
$$

because $T_{k}$ now takes the value $n+1$ on $F_{i}$ for each $i$, and it takes the value $i$ on $E_{i}$ as in model 1 (i.e. the possession of the candidate is terminated only by the arrival of a new candidate). Comparing (2.4) and (2.8) yields

$$
S_{2}(k)=S_{1}(k)+\sum_{i=k+1}^{n+1}(n+1-i) \mathbb{P}\left\{F_{i} \mid N \geq k\right\},
$$


or, equivalently, from (2.6),

$$
\pi_{k} S_{2}(k)=\pi_{k} S_{1}(k)+\sum_{i=k}^{n}(n-i) B_{k, i} p_{i},
$$

which, combined with (2.7), gives (2.3). This completes the proof.

Remark 2.1. It may be more instructive to express the duration in terms of the indicators $X_{j}$ and $N$. Let $D(k)\left(=T_{k}-k\right)$ denote the duration or payoff when we stop in state $k$. Then, setting $Y_{j}=1-X_{j}$, we easily find that, for $1 \leq k \leq n$,

$$
\begin{aligned}
& D(k)=1+\sum_{i=k+1}^{N} \prod_{j=k+1}^{i} Y_{j} \quad \text { for model 1, } \\
& D(k)=1+\sum_{i=k+1}^{N} \prod_{j=k+1}^{i} Y_{j}+(n-N) \prod_{j=k+1}^{N} Y_{j} \quad \text { for model } 2 .
\end{aligned}
$$

Thus, we can also obtain $S(k)$ as $\mathbb{E}[D(k) \mid N \geq k]$ by a straightforward calculation.

Remark 2.2. Suppose that one is to choose a partner in life and is considered satisfied with his/her choice so long as the partner chosen stays relatively best. Model 1 depicts a situation in which the lifetime of the chooser is $N$ and the opportunities to choose are available all through his/her life, while model 2 depicts a situation in which the lifetime of the chooser is $n$, but the opportunities to choose are available only in his/her youth of random length $N$.

If we decide not to stop in state $k(<n)$ and proceed to the next stage, we can observe the $(k+1)$ th object with probability $\pi_{k+1} / \pi_{k}$, but observe no object with the remaining probability $1-\pi_{k+1} / \pi_{k}$, in which case we come to know that the $k$ th object was the last object, and so we have failed to choose an object. The $(k+1)$ th object, if observed, results in a candidate or a noncandidate with respective probabilities of $a_{k+1}$ and $b_{k+1}$. Hence, we have

$$
C(k)=\frac{\pi_{k+1}}{\pi_{k}}\left[a_{k+1} V(k+1)+b_{k+1} C(k+1)\right], \quad 1 \leq k<n,
$$

with the obvious boundary condition $C(n)=0$.

It is easy to see that repeated use of (2.9) yields

$$
C(k)=\frac{1}{\pi_{k}} \sum_{j=k+1}^{n} \pi_{j} B_{k, j} r_{j} V(j)
$$

if we let $r_{j}=a_{j} / b_{j}, 1 \leq j \leq n$. Define $\tilde{C}(k)$ to be the expected payoff by using a rule of stopping with the first candidate after leaving state $k$, if any. Then, from (2.10),

$$
\tilde{C}(k)=\frac{1}{\pi_{k}} \sum_{j=k+1}^{n} \pi_{j} B_{k, j} r_{j} S(j),
$$

which is written as follows from Lemma 2.1. 
Lemma 2.2. For $1 \leq k<n$,

$$
\begin{aligned}
& \tilde{C}(k)=\frac{1}{\pi_{k}} \sum_{i=k+1}^{n}\left(\sum_{j=k+1}^{i} r_{j}\right) \pi_{i} B_{k, i} \text { for model } 1, \\
& \tilde{C}(k)=\frac{1}{\pi_{k}} \sum_{i=k+1}^{n}\left(\sum_{j=k+1}^{i} r_{j}\right) \sigma_{i} B_{k, i} \quad \text { for model } 2 .
\end{aligned}
$$

Now let

$$
t(n)=\min \{j: S(k) \geq \tilde{C}(k) \text { for all } j \leq k \leq n\} .
$$

Then backward induction shows that it is optimal to stop in state $k \geq t(n)$. A problem of interest is to compare $t(n)$ with the threshold value $s^{*}(n)$ given below in (2.13). As a generalization of the classical best-choice problem, Bruss (2000) considered the problem of stopping with the last candidate in the setting of Bernoulli trials of given length $n$. The main result can be described as follows.

Theorem 2.1. (Bruss' odds theorem.) For the problem of maximizing the probability of stopping with the last candidate in $n$ Bernoulli trials (i.e. $N$ is degenerate to $n$ ), the optimal rule is simple, i.e. it stops with the first candidate $X_{k}=1$ with $k \geq s^{*}(n)$, if any, where

$$
s^{*}(n)=\min \left\{k \geq 1: \sum_{j=k+1}^{n} r_{j} \leq 1\right\} .
$$

The classical best-choice problem appears in the secretary case, because the last candidate is the very best in this case $\left((2.13)\right.$ is reduced to $s_{c}^{*}(n)$ in (1.1)). Ferguson (2008) and Tamaki (2010), (2011) further generalized Bruss' odds theorem. The following lemma shows that, in the RHDP, we stop earlier than in the corresponding Bruss problem.

Lemma 2.3. Let $n$ be fixed. Then, whatever the distribution of $N(\leq n)$ might be, the first time the optimal rule will stop with a candidate occurs no later than the $s^{*}(n)$ th trial, where $s^{*}(n)$ is as defined in (2.13). Moreover, the optimal rule stops with the first candidate among trials $s^{*}(n), s^{*}(n)+1, \ldots, n$ if stopping has not occurred previously.

Proof. It suffices to show that $t(n) \leq s^{*}(n)$. From Lemmas 2.1 and $2.2, S(k) \geq \tilde{C}(k)$ is equivalent to

$$
\begin{aligned}
& \pi_{k}+\sum_{i=k+1}^{n}\left(1-\sum_{j=k+1}^{i} r_{j}\right) \pi_{i} B_{k, i} \geq 0 \quad \text { for model 1, } \\
& \sigma_{k}+\sum_{i=k+1}^{n}\left(1-\sum_{j=k+1}^{i} r_{j}\right) \sigma_{i} B_{k, i} \geq 0 \quad \text { for model 2, }
\end{aligned}
$$

implying that $t(n) \leq s^{*}(n)$ from (2.12) and (2.13), because $r_{j}$ is nonnegative.

In contrast to the Bruss problem, the optimal rule of the RHDP is not necessarily simple, even if $N$ is degenerate. Assume that $N$ degenerates to $n$. The corresponding optimality equation is, from (2.2) and (2.10),

$$
V(k)=\max \left\{\sum_{j=k}^{n} B_{k, j}, \sum_{j=k+1}^{n} B_{k, j} r_{j} V(j)\right\}, \quad 1 \leq k<n,
$$


with $V(n)=1$ for $n \geq 2$. The optimal rule depends on the probability sequence $\left(b_{2}, b_{3}, \ldots, b_{n}\right)$ and not on $b_{1}$. A bit of calculation from (2.15) shows that the optimal rule is always simple for $n=2,3$ but not for $n=4$. In fact, for $n=4$, the optimal stopping region, defined as the set of states for which stopping is optimal, is $\{1,3,4\}$, if and only if $\left(b_{2}, b_{3}, b_{4}\right)$ satisfies the set of inequalities

$$
b_{2}>\frac{\left(1-2 b_{3}\right) b_{4}}{1+\left(1+b_{4}\right) b_{3}}, \quad b_{3}<\frac{1}{4}, \quad b_{4}>\frac{b_{3}}{1-3 b_{3}} .
$$

For this example, we can give $\left(b_{2}, b_{3}, b_{4}\right)=\left(\frac{2}{5}, \frac{1}{5}, \frac{3}{5}\right)$. A similar phenomenon is true for $n \geq 5$, and also for random $N$. So, in the next two sections, we seek, for each model, a sufficient condition for the optimal rule to be simple for $n \geq 4$.

Remark 2.3. The aim of the classical best-choice problem is to choose the very best. If the aim is generalized to choose an object whose rank belongs to a prescribed set of ranks, the optimal rule becomes very complicated, and not always simple. See, e.g. Suchwalko and Szajowski (2002).

\section{Model 1}

We deal with model 1 in this section. Make the following transformations:

$$
\begin{aligned}
s(k) & =B_{1, k} \pi_{k} S(k), \\
c(k) & =B_{1, k} \pi_{k} C(k), \\
v(k) & =B_{1, k} \pi_{k} V(k)=\max \{s(k), c(k)\}, \\
\mu(k) & =s(k)-c(k) .
\end{aligned}
$$

Then we have, from (2.2) and (2.10),

$$
s(k)=s(k+1)+\pi_{k} B_{1, k}
$$

and

$$
c(k)=c(k+1)+r_{k+1} v(k+1),
$$

respectively. Subtracting (3.6) from (3.5) on both sides yields

$$
\mu(k)=\mu(k+1)+\pi_{k} B_{1, k}-r_{k+1} v(k+1),
$$

or, equivalently,

$$
\mu(k)=\mu(k+1)+r_{k+1} \min \{0, \mu(k+1)\}+B_{1, k} G(k)
$$

with $\mu(n)=B_{1, n} p_{n}$, if we define a function

$$
G(k)=\pi_{k}-r_{k+1} \sum_{i=k+1}^{n} \pi_{i} B_{k, i}, \quad 1 \leq k<n .
$$

Then we have the following result.

Theorem 3.1. A sufficient condition for the optimal rule to be simple is that $G(k)$ changes its sign from - to + at most once before $s^{*}(n)$, that is,

$$
\text { once } G(k) \geq 0 \text { for some } k \text {, then } G(j) \geq 0 \text { for all } k \leq j<s^{*}(n) \text {. }
$$


Proof. We have $\mu(k) \geq 0$ for $k \geq s^{*}(n)$ from Lemma 2.3. Hence, to establish the optimality of the threshold rule, it suffices to show that $\mu(k)$ is unimodal for $k<s^{*}(n)$. We omit the proof because it is similar to that of Theorem 2.2 of Tamaki (2011) for the probability maximization problem (see also Petruccelli (1983), who showed that $\mu(k)$, defined by (3.7), is unimodal in the secretary case if $G(k)$ satisfies condition (3.9)).

For later use, it should be noted that $G(k)$ in (3.8) satisfies the recursion

$$
G(k)=\frac{a_{k+1} b_{k+2}}{a_{k+2}} G(k+1)+\left(\pi_{k}-\frac{a_{k+1}}{a_{k+2}} \pi_{k+1}\right)
$$

for $k<n-1$ with $G(n-1)=\pi_{n-1}-a_{n} \pi_{n}=p_{n-1}+b_{n} p_{n}>0$.

If the optimal rule turns out to be simple, the (optimal) threshold value is given as, from (2.14),

$$
t^{*}(n)=\min \left\{k \geq 1: \sum_{i=k}^{n} \pi_{i} B_{k, i} \geq \sum_{i=k+1}^{n}\left(\sum_{j=k+1}^{i} r_{j}\right) \pi_{i} B_{k, i}\right\} .
$$

Observe that, from (3.7) and Theorem 3.1, if $G(k) \geq 0$ for all $k<s^{*}(n)$, the optimal rule is simple with threshold value $t^{*}(n)=1$, that is, it stops with the first candidate.

The expected payoff under the simple rule with threshold value $t$ is calculated as

$$
\Phi(n, t)=\pi_{t-1} \tilde{C}(t-1)=\sum_{i=t}^{n}\left(\sum_{j=t}^{i} r_{j}\right) \pi_{i} B_{t-1, i}
$$

from (2.11), because our payoff is $\tilde{C}(t-1)$ by pretending we are leaving state $t-1$ if the Bernoulli trials continue up to time $t-1$; otherwise, our payoff is 0 . Moreover, the proportional payoff is defined as $\Psi(n, t)=\Phi(n, t) / n$, to make the solution more easily comparable to other problems. The optimal payoff and the optimal proportional payoff are then denoted by $\Phi^{*}(n)=\Phi\left(n, t^{*}(n)\right)$ and $\Psi^{*}(n)=\Psi\left(n, t^{*}(n)\right)$, respectively.

Corollary 3.1. Let $N$ be degenerate. Then if $S(k)=\sum_{i=k}^{n} B_{k, i}$ is unimodal in the sense that, for some finite integer $K, S(k) \leq S(k+1)$ for $k<K$ and $S(k) \geq S(k+1)$ for $k \geq K$, the optimal rule is simple.

Proof. Since $N$ is degenerate, we have, from (2.2) and (3.8), $S(k)=\sum_{i=k}^{n} B_{k, i}$ and

$$
G(k)=1-r_{k+1} \sum_{i=k+1}^{n} B_{k, i}=S(k)-S(k+1) .
$$

Condition (3.9) now implies that $S(k)$ should be unimodal. This completes the proof.

Corollary 3.1 can be seen as a direct consequence of Lemma 2.1 of Ferguson et al. (1992), because $\sum_{j=k+1}^{n} B_{k, j} r_{j} V(j)(=C(k))$ in (2.15) is shown to be nonincreasing in $k$. For the purposes of most applications to the secretary case, the following corollary is useful.

Corollary 3.2. Let, for $1 \leq k<n-1$,

$$
H(k)=(k+1) \pi_{k}-(k+2) \pi_{k+1}=(k+1) p_{k}-\pi_{k+1} .
$$

Then, in the secretary case, a sufficient condition for the optimal rule to be simple is that $H(k)$ changes its sign from - to + at most once. 
Proof. In the secretary case, (3.10) is reduced to

$$
G(k)-G(k+1)=\frac{1}{k+1} H(k) .
$$

Thus, condition (3.9) is assured, because $G(k)$ is unimodal (with $G(n-1)>0$ ), if $H(k)$ satisfies the stated condition.

Corollary 3.2 is applicable to the following distributions. In the first three examples, $H(k)$ is nondecreasing in $k$.

Example 3.1. ( $N$ degenerates to $n$.) Here $p_{n}=1$ and $p_{k}=0,1 \leq k<n$. We have $H(k) \equiv$ -1. This is the classical duration problem mentioned in Section 1 . Since $r_{j}=1 /(j-1)$, (3.11) is reduced to $t_{c}^{*}(n)$ in (1.2). The asymptotic results $t_{c}^{*}(n) / n \rightarrow \mathrm{e}^{-2}$ and $\Psi^{*}(n) \rightarrow 2 \mathrm{e}^{-2}$ as $n \rightarrow \infty$ are easily obtained from (3.11) and (3.12).

Example 3.2. (Uniform.) Here $p_{k}=1 / n, 1 \leq k \leq n$. We have $H(k)=(2 k+1-n) / n$.

Example 3.3. (Generalized uniform.) Here

$$
p_{k}= \begin{cases}0 & \text { if } 1 \leq k<T, \\ \frac{1}{n-T+1} & \text { if } T \leq k \leq n,\end{cases}
$$

for a given parameter $T=1,2, \ldots, n$. We have

$$
H(k)= \begin{cases}-1 & \text { if } k<T, \\ \frac{2 k+1-n}{n-T+1} & \text { if } k \geq T .\end{cases}
$$

The cases $T=n$ and $T=1$ correspond to Example 3.1 and Example 3.2, respectively. Hence, this example serves as a unification of the first two examples.

Example 3.4. (Curtailed geometric.) Here $p_{k}=(1-q) q^{k-1} /\left(1-q^{n}\right), 1 \leq k \leq n$, for a given parameter $0<q<1$. A bit of calculation gives

$$
H(k)=\frac{q^{k-1}}{1-q^{n}} K(k),
$$

where

$$
K(k)=(1-q)(k+1)-q\left(1-q^{n-k}\right) .
$$

Since $K(k)$ is increasing in $k, H(k)$ changes its sign from - to + at most once.

The asymptotics in Examples 3.2, 3.3, and 3.4 as $n \rightarrow \infty$ will be examined in Section 3.1 in detail. Besides the secretary case, a problem of interest may include the case of the constant occurrence probability of a candidate, i.e. $a_{j} \equiv a$. As is intuitively clear, the optimal rule now stops with the first candidate. This remarkable feature can be ascertained as follows.

Corollary 3.3. Assume that $a_{j} \equiv a$, i.e. $b_{j} \equiv b=1-a$. Then the optimal rule is simple with threshold value 1 , whatever the distribution of $N$ might be.

Proof. It is sufficient to show that $G(k)>0$ for all $k<n$. This can be shown inductively if we use, starting with $G(n-1)=p_{n-1}+b p_{n}>0$, the relation

$$
G(k)=b G(k+1)+p_{k}
$$

obtained from (3.10). 


\subsection{Asymptotic results in the secretary case}

For Examples 3.2, 3.3, and 3.4, we can obtain the limiting values

$$
z^{*}=\lim _{n \rightarrow \infty} \frac{t^{*}(n)}{n}, \quad v^{*}=\lim _{n \rightarrow \infty} \Psi^{*}(n)
$$

by letting the prior distribution depend on $n$. Since $r_{j}=1 /(j-1)$ and $B_{t-1, i}=(t-1) / i$ in the secretary case, we have, from (3.12),

$$
\Psi(n, t)=\frac{t-1}{n} \sum_{i=t}^{n}\left(\sum_{j=t}^{i} \frac{1}{j-1}\right) \frac{\pi_{i}}{i} .
$$

Substituting $\pi_{i}=\sum_{k=i}^{n} p_{k}$ into (3.13) yields another expression

$$
\Psi(n, t)=\frac{t-1}{n} \sum_{k=t}^{n}\left(\sum_{j=t}^{k} \frac{1}{j-1} \sum_{i=j}^{k} \frac{1}{i}\right) p_{k} .
$$

For the uniform distribution of Example 3.2, $p_{k}=1 / n$, so (3.14) is

$$
\Psi_{\mathrm{U}}(n, t)=\frac{t-1}{n} \sum_{k=t}^{n}\left(\sum_{j=t}^{k} \frac{1}{j-1} \sum_{i=j}^{k} \frac{1}{i}\right) \frac{1}{n} .
$$

If we let $n$ tend to $\infty$ and write $z$ as the limit of $t / n$, then, using $x$ for $i / n, y$ for $j / n, v$ for $k / n$, and $\mathrm{d} x, \mathrm{~d} y$, and $\mathrm{d} v$ for $1 / n, \Psi_{\mathrm{U}}(n, t)$ becomes a Riemann approximation to an integral, i.e. $\Psi_{\mathrm{U}}(n, t) \rightarrow \psi_{\mathrm{U}}(z)$, where

$$
\begin{aligned}
\psi_{\mathrm{U}}(z) & =z \int_{z}^{1}\left(\int_{z}^{v} \frac{\mathrm{d} y}{y} \int_{y}^{v} \frac{\mathrm{d} x}{x}\right) \mathrm{d} v \\
& =\frac{z}{2} \int_{z}^{1} \log ^{2}\left(\frac{v}{z}\right) \mathrm{d} v \\
& =z\left(1-z+\log z+\frac{1}{2} \log ^{2} z\right) .
\end{aligned}
$$

We now have the following results.

Lemma 3.1. (Uniform prior.) Let $\alpha^{*}(\approx 0.07754)$ be the unique root $\alpha \in(0,1)$ of the equation

$$
2(1+\sqrt{\alpha})+\log \alpha=0
$$

Then

$$
\begin{aligned}
& z^{*}=\alpha^{*} \\
& v^{*}=\alpha^{*}\left(1+\sqrt{\alpha^{*}}\right)^{2} \approx 0.12674
\end{aligned}
$$

Proof. The value $z$ that maximizes $\psi_{\mathrm{U}}(z)$ in (3.15) is found by setting the derivative with respect to $z$ equal to 0 and then solving for $z$. When this is done, we have

$$
\psi_{\mathrm{U}}^{\prime}(z)=\frac{1}{2}[2(1+\sqrt{z})+\log z][2(1-\sqrt{z})+\log z],
$$


implying that $\psi_{\mathrm{U}}^{\prime}(z)=0$ for $z \in(0,1)$ is equivalent to $2(1+\sqrt{z})+\log z=0$, because we easily find that

$$
2(1-\sqrt{z})+\log z<0, \quad 0<z<1 .
$$

Thus, we have established (3.17) since the optimal $z \in(0,1)$ can be considered as $\lim _{n \rightarrow \infty} t^{*}(n) / n$ from the above approximation. Substituting the optimal value $z^{*}=\alpha^{*}$ back into (3.15) and then using (3.16) yields the desired optimal payoff $\psi_{\mathrm{U}}\left(\alpha^{*}\right)$ as given in (3.18). This completes the proof.

Remark 3.1. Let us say that the random horizon is triangular if the prior distribution of $N$ is given by

$$
p_{k}=\frac{2(n-k+1)}{n(n+1)}, \quad 1 \leq k \leq n .
$$

Gnedin (2005, Section 5) showed that model 1 with $N$ uniform on $\{1, \ldots, n\}$ is equivalent to the best-choice problem with triangular random horizon in a sense. This implies from Lemma 3.1 combined with Proposition 4.1 of Gnedin (2005) that, as $n \rightarrow \infty$, the latter problem has the same threshold value $z^{*} \approx 0.07754$ and corresponding probability $2 v^{*} \approx 0.25348$. Note that, for a finite $n$, the threshold value and the corresponding probability for the best-choice problem with random horizon can be calculated from Equations (2.26) and (2.27) of Tamaki (2011), respectively, by putting $m=1$.

In a similar manner, we can deal with other examples. Assume that $n p_{k} \rightarrow f(v)$ for some bounded function $f(v)$ if we let $n \rightarrow \infty$ and $k \rightarrow \infty$ in such a way that $k / n \rightarrow v$. Then $\Psi(n, t)$ in (3.14) becomes an approximation to

$$
\psi(z)=z \int_{z}^{1}\left(\int_{z}^{v} \frac{\mathrm{d} y}{y} \int_{y}^{v} \frac{\mathrm{d} x}{x}\right) f(v) \mathrm{d} v=\frac{z}{2} \int_{z}^{1} \log ^{2}\left(\frac{v}{z}\right) f(v) \mathrm{d} v .
$$

The function $f(v)$ can be viewed as a density function of the random truncation time $V(\leq 1)$ adjusted to $N ; \psi_{\mathrm{U}}(z)$ in (3.15) corresponds to $f(v) \equiv 1$.

Lemma 3.2. (Generalized uniform prior.) Let $T$ depend on $n$ in such a way that $T / n \rightarrow \alpha$ as $n \rightarrow \infty$ for a fixed $0<\alpha<1$. Then the asymptotic results are distinguished between two cases according to whether $\alpha \leq \alpha^{*}$ or $\alpha>\alpha^{*}$, where $\alpha^{*}$ is as defined in Lemma 3.1 (below we use the notation $z_{\alpha}^{*}$ and $v_{\alpha}^{*}$ for $z^{*}$ and $v^{*}$, respectively, to make explicit the dependence on $\alpha)$.

(i) For $0 \leq \alpha \leq \alpha^{*}$,

$$
\begin{aligned}
& z_{\alpha}^{*}=\alpha^{*}, \\
& v_{\alpha}^{*}=\frac{\alpha^{*}\left(1+\sqrt{\alpha^{*}}\right)^{2}}{1-\alpha} .
\end{aligned}
$$

(ii) $\operatorname{For} \alpha^{*}<\alpha<1$,

$$
\begin{aligned}
& z_{\alpha}^{*}=\alpha^{(\sqrt{\alpha}-\alpha) /(1-\alpha)} \mathrm{e}^{-2}, \\
& v_{\alpha}^{*}=\left(1-\frac{\sqrt{\alpha}}{1-\alpha} \log \alpha\right) z_{\alpha}^{*}
\end{aligned}
$$

Proof. Obviously,

$$
f(v)= \begin{cases}0 & \text { if } 0<v \leq \alpha \\ \frac{1}{1-\alpha} & \text { if } \alpha<v<1\end{cases}
$$


Write $\psi_{\alpha}(z)$ for $\psi(z)$ to show dependence on $\alpha$. Then a straightforward calculation from (3.20) leads to

$$
\begin{aligned}
\psi_{\alpha}(z) & =\frac{z}{2(1-\alpha)} \int_{\max (\alpha, z)}^{1} \log ^{2}\left(\frac{v}{z}\right) \mathrm{d} v \\
& = \begin{cases}\frac{z}{2(1-\alpha)}\left[1-2 z+\{p(z)+1\}^{2}\right] & \text { if } \alpha<z, \\
\frac{z}{2(1-\alpha)}\left[1-\alpha+\{p(z)+1\}^{2}-\alpha\left\{q_{\alpha}(z)+1\right\}^{2}\right] & \text { if } z \leq \alpha,\end{cases}
\end{aligned}
$$

where

$$
\begin{gathered}
p(z)=\log \left(\frac{1}{z}\right)-2, \quad 0<z<1, \\
q_{\alpha}(z)=p(z)-\log \left(\frac{1}{\alpha}\right), \quad 0<\alpha<1 .
\end{gathered}
$$

Differentiating $\psi_{\alpha}(z)$ with respect to $z$ yields

$$
\psi_{\alpha}^{\prime}(z)= \begin{cases}\frac{1}{2(1-\alpha)}[p(z)-2 \sqrt{z}][p(z)+2 \sqrt{z}] & \text { if } \alpha<z, \\ \frac{1}{2(1-\alpha)}\left[p(z)+\sqrt{\alpha} q_{\alpha}(z)\right]\left[p(z)-\sqrt{\alpha} q_{\alpha}(z)\right] & \text { if } z \leq \alpha .\end{cases}
$$

We can show the following inequalities:

(a) $p(z)+2 \sqrt{z}>0,0<z<1$,

(b) $p(z)-\sqrt{\alpha} q_{\alpha}(z)>0,0<z<\alpha<1$.

Inequality (a) is just the inequality given in (3.19). As for (b), we have, from (a),

$$
p(\alpha)+2 \sqrt{\alpha}>0, \quad 0<\alpha<1,
$$

or, equivalently,

$$
-p(\alpha)<\frac{\sqrt{\alpha}}{1-\sqrt{\alpha}}[p(\alpha)+2] .
$$

Applying $-p(z)<-p(\alpha)$ for $z<\alpha$ to the left-hand side of this inequality gives (b). Hence, the sign of $\psi_{\alpha}^{\prime}(z)$ coincides with that of the function $\eta_{\alpha}(z)$ defined as

$$
\eta_{\alpha}(z)= \begin{cases}p(z)-2 \sqrt{z} & \text { if } \alpha<z, \\ p(z)+\sqrt{\alpha} q_{\alpha}(z) & \text { if } z \leq \alpha .\end{cases}
$$

Therefore, to derive (3.21) and (3.23), it is sufficient to show that

1. $\eta_{\alpha}(z)=0$ has a unique root $z \in(0,1)$ for each $\alpha$,

2. $\eta_{\alpha}(z)=0$ is reduced to $p(z)-2 \sqrt{z}=0$ for $\alpha \leq \alpha^{*}$, whereas $\eta_{\alpha}(z)=0$ is reduced to $p(z)+\sqrt{\alpha} q_{\alpha}(z)=0$, or, equivalently, $p(z)=-\sqrt{\alpha} \log (\alpha) /(1+\sqrt{\alpha})$ for $\alpha>\alpha^{*}$.

Assertion 1 is immediate because $\eta_{\alpha}(z)$ is a continuous and decreasing function of $z$ with boundary conditions $\lim _{z \rightarrow 0} \eta_{\alpha}(z)=\infty$ and $\lim _{z \rightarrow 1} \eta_{\alpha}(z)=-4$. For assertion 2 , it is important to note that $\eta_{\alpha}(\alpha)=p(\alpha)-2 \sqrt{\alpha}$ is decreasing in $\alpha$ such that $\eta_{\alpha}(\alpha) \geq 0$ if $\alpha \leq \alpha^{*}$, 
TABLE 1: Values of $z_{\alpha}^{*}$ and $v_{\alpha}^{*}$ for several $\alpha$ (generalized uniform prior).

\begin{tabular}{cccccc}
\hline \multirow{2}{*}{$\alpha$} & \multicolumn{2}{c}{ Model 1 } & & \multicolumn{2}{c}{ Model 2 } \\
\cline { 2 - 3 } \cline { 5 - 6 } & $z_{\alpha}^{*}$ & $v_{\alpha}^{*}$ & & $z_{\alpha}^{*}$ & $v_{\alpha}^{*}$ \\
\hline 0.0 & 0.07754 & 0.12674 & & 0.07754 & 0.25348 \\
0.1 & 0.07783 & 0.14080 & & 0.08554 & 0.27999 \\
0.2 & 0.08230 & 0.15635 & & 0.10874 & 0.28632 \\
0.3 & 0.08838 & 0.17165 & & 0.12124 & 0.28441 \\
0.4 & 0.09489 & 0.18655 & & 0.12819 & 0.28102 \\
0.5 & 0.10156 & 0.20111 & & 0.13197 & 0.27774 \\
0.6 & 0.10829 & 0.21541 & & 0.13392 & 0.27504 \\
0.7 & 0.11504 & 0.22947 & & 0.13484 & 0.27302 \\
0.8 & 0.12180 & 0.24335 & & 0.13521 & 0.27167 \\
0.9 & 0.12857 & 0.25708 & & 0.13532 & 0.27091 \\
1.0 & 0.13534 & 0.27067 & & 0.13534 & 0.27067 \\
\hline
\end{tabular}

whilst $\eta_{\alpha}(\alpha)<0$ if $\alpha>\alpha^{*}$ ( $\alpha^{*}$ is the unique root of the equation $\eta_{\alpha}(\alpha)=0$ ). This just proves assertion 2 because, if $\alpha \leq \alpha^{*}$, $z_{\alpha}^{*}$ must be greater than or equal to $\alpha$, whereas, if $\alpha>\alpha^{*}, z_{\alpha}^{*}$ must be less than $\alpha$. The $v_{\alpha}^{*}$ term in (3.22) and (3.24) is obtained from (3.26) through $v_{\alpha}^{*}=\psi_{\alpha}\left(z_{\alpha}^{*}\right)$, combined with the optimization condition $\eta_{\alpha}\left(z_{\alpha}^{*}\right)=0$. This completes the proof.

We list the values of $z_{\alpha}^{*}$ and $v_{\alpha}^{*}$ for some $\alpha$ in Table 1 together with the corresponding values for model 2 (see Section 4). Inspection of this table shows that both $z_{\alpha}^{*}$ and $v_{\alpha}^{*}$ are increasing in $\alpha$ (except for $z_{\alpha}^{*} \equiv \alpha^{*}$ for $0 \leq \alpha \leq \alpha^{*}$ ).

To examine a general property of $\psi(z)$ in (3.20), differentiate $\psi(z)$ with respect to $z$. Then

$$
\begin{aligned}
\psi^{\prime}(z) & =\frac{1}{2}\left[\int_{z}^{1} \log ^{2}\left(\frac{v}{z}\right) f(v) \mathrm{d} v+z \int_{z}^{1} \frac{\partial}{\partial z}\left\{\log ^{2}\left(\frac{v}{z}\right)\right\} f(v) \mathrm{d} v\right] \\
& =\frac{1}{z} \psi(z)-\int_{z}^{1} \log \left(\frac{v}{z}\right) f(v) \mathrm{d} v .
\end{aligned}
$$

Thus, the optimal $z\left(=z^{*}\right)$ satisfies $\psi^{\prime}(z)=0$, or, equivalently,

$$
\int_{z}^{1} \log \left(\frac{v}{z}\right)\left[\log \left(\frac{v}{z}\right)-2\right] f(v) \mathrm{d} v=0,
$$

implying that $z^{*} \leq \mathrm{e}^{-2}$, because $\log (v / z)<2$ for $\mathrm{e}^{-2}<z<v<1$. The optimal payoff is given by (from (3.29))

$$
v^{*}=\psi\left(z^{*}\right)=z^{*} \int_{z^{*}}^{1} \log \left(\frac{v}{z^{*}}\right) f(v) \mathrm{d} v .
$$

To obtain interesting asymptotic results for the curtailed geometric prior given in Example 3.4, we allow the parameter $q$ to depend on $n$.

Lemma 3.3. (Curtailed geometric prior.) Let $q$ depend on $n$ through $q=1-c / n$ for a fixed positive value $c(<n)$. We use the notation $z_{c}^{*}$ and $v_{c}^{*}$ for $z^{*}$ and $v^{*}$, respectively, to make 
TABLE 2: Values of $z_{c}^{*}$ and $v_{c}^{*}$ for several $c$ (curtailed geometric prior).

\begin{tabular}{cccccc}
\hline \multirow{2}{*}{$c$} & \multicolumn{2}{c}{ Model 1 } & & \multicolumn{2}{c}{ Model 2 } \\
\cline { 2 - 3 } \cline { 5 - 6 } & $z_{c}^{*}$ & $v_{c}^{*}$ & & $z_{c}^{*}$ & $v_{c}^{*}$ \\
\hline 0 & 0.07754 & 0.12674 & & 0.07754 & 0.25348 \\
0.01 & 0.07745 & 0.12651 & & 0.07740 & 0.25341 \\
0.1 & 0.07656 & 0.12440 & & 0.07608 & 0.25279 \\
0.5 & 0.07257 & 0.11514 & & 0.07029 & 0.25007 \\
1 & 0.06747 & 0.10396 & & 0.06332 & 0.24688 \\
2 & 0.05731 & 0.08381 & & 0.05096 & 0.24163 \\
3 & 0.04793 & 0.06743 & & 0.04126 & 0.23811 \\
4 & 0.03995 & 0.05484 & & 0.03403 & 0.23600 \\
5 & 0.03355 & 0.04543 & & 0.02871 & 0.23481 \\
10 & 0.01740 & 0.02328 & & 0.01580 & 0.23329 \\
20 & 0.00870 & 0.01164 & & 0.00828 & 0.23298 \\
30 & 0.00600 & 0.00803 & & 0.00580 & 0.23292 \\
\hline
\end{tabular}

explicit the dependence on $c$. Then $z_{c}^{*}$ is a unique root $z \in(0,1)$ of the equation

$$
J_{c}(z)+\log z\left[I_{c}(z)+\mathrm{e}^{-c}\left(1+\frac{1}{2} \log z\right)\right]=0,
$$

where

$$
I_{c}(z)=\int_{z}^{1} \frac{\mathrm{e}^{-c x}}{x} \mathrm{~d} x, \quad J_{c}(z)=\int_{z}^{1} \frac{\mathrm{e}^{-c x}}{x}(1-\log x) \mathrm{d} x .
$$

Moreover,

$$
v_{c}^{*}=\frac{z_{c}^{*}}{1-\mathrm{e}^{-c}}\left[\mathrm{e}^{-c} \log z_{c}^{*}+I_{c}\left(z_{c}^{*}\right)\right] .
$$

Proof. Letting $n \rightarrow \infty$ and $k / n \rightarrow v$, we find that

$$
n p_{k}=\frac{c(1-c / n)^{k-1}}{1-(1-c / n)^{n}} \rightarrow f(v)=\frac{c \mathrm{e}^{-c v}}{1-\mathrm{e}^{-c}} .
$$

Thus, in this case, $f(v)$ turns out to be curtailed exponential with parameter $c$, and so (3.32) and (3.33) are obtained by straightforward calculations from (3.30) and (3.31), respectively.

We list the values of $z_{c}^{*}$ and $v_{c}^{*}$ for some $c$ in Table 2 together with the corresponding values for model 2 (see Section 4). Inspection of this table shows that both $z_{c}^{*}$ and $v_{c}^{*}$ are decreasing in $c$. Denote by $V_{c}$ the truncation time in the above lemma. Then its expected value is given by

$$
\mathbb{E}\left[V_{c}\right]=\int_{0}^{1} v f(v) \mathrm{d} v=\frac{\mathrm{e}^{c}-c-1}{c\left(\mathrm{e}^{c}-1\right)} .
$$

Since $\mathbb{E}\left[V_{c}\right] \rightarrow 0$ as $c \rightarrow \infty$, both $z_{c}^{*}$ and $v_{c}^{*}$ tend to 0 as $c \rightarrow \infty$. On the other hand, as $c \rightarrow 0, V_{c}$ converges in distribution to the uniform distribution on $(0,1)$ (consistent with $\left.\lim _{c \rightarrow 0} \mathbb{E}\left[V_{c}\right]=\frac{1}{2}\right)$.

Remark 3.2. The asymptotic argument given in this section is rather intuitive. We can make it more rigorous by approximating the difference equation (2.9) by a differential equation. This method was suggested in Dynkin and Yushkevich (1969). Mucci (1973a), (1973b) developed the idea for a wider class of optimal stopping problems. 


\section{Model 2}

In this section we consider model 2. We make the transformations (3.1)-(3.4) here as well. Then we see that, though (3.5) must be replaced by

$$
s(k)=s(k+1)+\sigma_{k} B_{1, k}
$$

from (2.3), (3.7) still holds if $G(k)$ is newly defined as

$$
G(k)=\sigma_{k}-r_{k+1} \sum_{i=k+1}^{n} \sigma_{i} B_{k, i}, \quad 1 \leq k<n .
$$

Analogously to Theorem 3.1, for model 2, we have the following result.

Theorem 4.1. A sufficient condition for the optimal rule to be simple is that $G(k)$ changes its sign from - to + at most once before $s^{*}(n)$.

Proof. The proof is similar to that of Theorem 3.1, and is thus omitted.

Remark 4.1. It is interesting to compare $G(\cdot)$-functions among three problems. Define, for $j=0,1,2$,

$$
G^{(j)}(k)=\alpha_{k}^{(j)}-r_{k+1} \sum_{i=k+1}^{n} \alpha_{i}^{(j)} B_{k, i}, \quad 1 \leq k<n,
$$

where $\alpha_{i}^{(0)}=p_{i}, \alpha_{i}^{(1)}=\pi_{i}$, and $\alpha_{i}^{(2)}=\sigma_{i}=\alpha_{i}^{(1)}+(n-i) \alpha_{i}^{(0)}$ for each $i$. The function $G^{(j)}(k)$ depends on the prior $\left\{p_{i}\right\}$ through $\left\{\alpha_{i}^{(j)}\right\}$. Theorems 3.1 and 4.1 show that $G(k)$ for model $j(j=1,2)$ is $G^{(j)}(k)$. Tamaki (2011) studied the problem of maximizing the probability of stopping with any of the last $m$ candidates in Bernoulli trials with random horizon. The $m=1$ case is considered as a random horizon version of the Bruss problem (see Bruss (2000)). Tamaki gave, in his Theorem 2.2, a similar sufficient condition for this problem to have a simple optimal rule, where the corresponding $G(k)$ is given by $G^{(0)}(k)$.

Analogously to (3.10), (3.11), and (3.12) for model 1, we can give the same expressions for model 2 by replacing $\pi$ with $\sigma$. That is,

$$
G(k)=\frac{a_{k+1} b_{k+2}}{a_{k+2}} G(k+1)+\left(\sigma_{k}-\frac{a_{k+1}}{a_{k+2}} \sigma_{k+1}\right)
$$

for $k<n-1$ with $G(n-1)=\sigma_{n-1}-a_{n} \sigma_{n}=2 p_{n-1}+b_{n} p_{n}>0$,

$$
t^{*}(n)=\min \left\{k \geq 1: \sum_{i=k}^{n} \sigma_{i} B_{k, i} \geq \sum_{i=k+1}^{n}\left(\sum_{j=k+1}^{i} r_{j}\right) \sigma_{i} B_{k, i}\right\}
$$

and

$$
\Phi(n, t)=\pi_{t-1} \tilde{C}(t-1)=\sum_{i=t}^{n}\left(\sum_{j=t}^{i} r_{j}\right) \sigma_{i} B_{t-1, i} .
$$

Of course, $t^{*}(n)$ and $\Phi(n, t)$ are defined as in model 1 .

For the applications to the secretary case, the following corollary is useful. 
Corollary 4.1. Let

$$
H(k)=(k+1) \sigma_{k}-(k+2) \sigma_{k+1}, \quad 1 \leq k<n-1 .
$$

Then, in the secretary case, a sufficient condition for the optimal rule to be simple is that $H(k)$ changes its sign from - to + at most once.

Proof. See the proof of Corollary 3.2, because (4.1) is reduced to

$$
G(k)-G(k+1)=\frac{1}{k+1} H(k) .
$$

Corollary 4.1 is applicable to the following distributions.

Example 4.1. (Uniform.) We have

$$
H(k)=\frac{2(2 k-n)+3}{n},
$$

which is increasing in $k$ and so satisfies the condition of Corollary 4.1.

Example 4.2. (Generalized uniform.) We have, for $1 \leq T \leq n$,

$$
H(k)= \begin{cases}-1 & \text { if } k \leq T-2, \\ -\frac{(n-T)(2+T)+1}{n-T+1} & \text { if } k=T-1, \\ \frac{2(2 k-n)+3}{n-T+1} & \text { if } k \geq T .\end{cases}
$$

It is easy to see that $H(k)$ satisfies the required condition, because $H(k)$ is increasing in $k(\geq T)$, attaining its bottom at $k=T-1$.

Example 4.3. (Curtailed geometric.) A bit of calculation gives

$$
H(k)=\frac{q^{k-1}}{1-q^{n}} K(k),
$$

where

$$
K(k)=(1-q)[(k+1)(n-k+1)-(k+2)(n-k-1) q]-q\left(1-q^{n-k}\right) .
$$

To show that $H(k)$ satisfies the condition of Corollary 4.1, it suffices to show that $K(k)$ is unimodal with $K(n-2)>0$ for $n \geq 4$. Let $L(k)=K(k)-K(k-1)$. Then, from (4.3),

$$
L(k)=(1-q)\left[(n-2 k+1)-(n-2 k-2) q+q^{n-k+1}\right],
$$

which implies that $L(k)$ is decreasing in $k$ because

$$
L(k)-L(k+1)=(1-q)^{2}\left(2-q^{n-k}\right)>0 .
$$

Thus, $K(k)$ proves to be unimodal. On the other hand, we again have, from (4.3),

$$
K(n-2)=(1-q)(3-q)\left[n-\frac{3+q(1+q)}{3-q}\right] .
$$

Since $(3+q(1+q)) /(3-q) \leq \frac{5}{2}, K(n-2)>0$ for $n \geq 4$. Thus, we have shown that $K(k)$ has the desired property. 
When it comes to the case of the constant occurrence probability, we obtain, from (4.1), the recursive relation

$$
G(k)=b G(k+1)+\left(\sigma_{k}-\sigma_{k+1}\right),
$$

and have the following results.

Corollary 4.2. Assume that $a_{j} \equiv$ a, i.e. $b_{j} \equiv b=1-a$. Then the following assertions hold.

(i) If $\sigma_{k}$ is unimodal, the optimal rule is simple.

(ii) If $\sigma_{k}$ is in particular nonincreasing in $k$, the optimal rule stops with the first candidate.

Proof. (i) Let $K$ be an integer such that $\sigma_{k}-\sigma_{k+1} \leq 0$ for $k<K$ and $\sigma_{k}-\sigma_{k+1} \geq 0$ for $k \geq K$. Since $G(n-1)>0$, we find that $G(k)>0$ for $k \geq K$ by induction from (4.4). This implies that if $G(k+1) \leq 0$ then $k \leq K-2$, and so $G(k) \leq 0$ again from (4.4), ensuring that $G(k)$ changes its sign from - to + at most once.

(ii) See the proof of Corollary 3.3. In a similar manner, we can show that $G(k)>0$ for all $k<n$.

Remark 4.2. Note that if $p_{k}$ is nonincreasing in $k, \sigma_{k}$ is also nonincreasing. Hence, the optimal rule with a uniform prior or a curtailed geometric prior stops with the first candidate from Corollary 4.2(ii). For a generalized uniform prior, the optimal rule is simple from Corollary 4.2(i). However, unlike model 1, it is not always the case that the optimal rule stops with the first candidate.

\subsection{Asymptotic results in the secretary case}

Let $\Psi(n, t)=\Phi(n, t) / n$. Then, in the secretary case, we have, from (4.2),

$$
\Psi(n, t)=\frac{t-1}{n} \sum_{k=t}^{n}\left(\sum_{j=t}^{k} \frac{1}{j-1} \sum_{i=j}^{k} \frac{1}{i}+\frac{n-k}{k} \sum_{j=t}^{k} \frac{1}{j-1}\right) p_{k} .
$$

It is easy to see that, if $n p_{k} \rightarrow f(v)$ for some bounded function $f(v)$ as $n \rightarrow \infty$ and $k \rightarrow \infty$ in such a way that $k / n \rightarrow v, \Psi(n, t)$ becomes an approximation to

$$
\begin{aligned}
\psi(z) & =z \int_{z}^{1}\left(\int_{z}^{v} \frac{\mathrm{d} y}{y} \int_{y}^{v} \frac{\mathrm{d} x}{x}+\frac{1-v}{v} \int_{z}^{v} \frac{\mathrm{d} y}{y}\right) f(v) \mathrm{d} v \\
& =\frac{z}{2} \int_{z}^{1} \log ^{2}\left(\frac{v}{z}\right) f(v) \mathrm{d} v+z \int_{z}^{1} \frac{1-v}{v} \log \left(\frac{v}{z}\right) f(v) \mathrm{d} v .
\end{aligned}
$$

The first term of (4.5) is just the expected payoff for model 1 (as given by (3.20)) and so the second term is the additional expected payoff. As before, let

$$
z^{*}=\lim _{n \rightarrow \infty} \frac{t^{*}(n)}{n}, \quad v^{*}=\lim _{n \rightarrow \infty} \Psi^{*}(n),
$$

where $\Psi^{*}(n)=\Psi\left(n, t^{*}(n)\right)$. For a uniform distribution, we have the following result.

Lemma 4.1. (Uniform prior.) Let $\alpha^{*}(\approx 0.07754)$ be as defined in Lemma 3.1. Then

$$
z^{*}=\alpha^{*}, \quad v^{*}=2 \alpha^{*}\left(1+\sqrt{\alpha^{*}}\right)^{2} \approx 0.25348 .
$$


Proof. Since $f(v) \equiv 1$, we have, from (4.5),

$$
\psi(z)=\frac{z}{2} \int_{z}^{1} \log ^{2}\left(\frac{v}{z}\right) \mathrm{d} v+z \int_{z}^{1} \frac{1-v}{v} \log \left(\frac{v}{z}\right) \mathrm{d} v .
$$

Straightforward calculations show that the first term and the second term are the same, i.e.

$$
\frac{z}{2} \int_{z}^{1} \log ^{2}\left(\frac{v}{z}\right) \mathrm{d} v=z \int_{z}^{1} \frac{1-v}{v} \log \left(\frac{v}{z}\right) \mathrm{d} v=z\left(1-z+\log z+\frac{1}{2} \log ^{2} z\right) .
$$

Therefore, we obtain

$$
\psi(z)=2 z\left(1-z+\log z+\frac{1}{2} \log ^{2} z\right),
$$

which yields the desired result by the same argument as used in the proof of Lemma 3.1.

It is interesting to see that, in comparison to model 1, the optimal threshold value is the same, but the optimal payoff is twice as large. This is not surprising because, in the uniform case, $\sigma_{i}=2 \pi_{i}-1 / n$ and so, from Lemma 2.1, the stopping payoff for model 2 is asymptotically two times as large as that for model 1.

For the generalized uniform prior, we have the following result.

Lemma 4.2. (Generalized uniform prior.) The two cases are distinguished according to whether $\alpha \leq \alpha^{*}$ or $\alpha>\alpha^{*}$, where $\alpha^{*}$ is as defined in Lemma 3.1 (below we use the notation $z_{\alpha}^{*}$ and $v_{\alpha}^{*}$ for $z^{*}$ and $v^{*}$, respectively).

(i) For $0 \leq \alpha \leq \alpha^{*}$,

$$
z_{\alpha}^{*}=\alpha^{*}, \quad v_{\alpha}^{*}=\frac{2 \alpha^{*}\left(1+\sqrt{\alpha^{*}}\right)^{2}}{1-\alpha}
$$

(ii) For $\alpha^{*}<\alpha<1$, let

$$
\rho=-\left(2+\frac{1+\alpha}{1-\alpha} \log \alpha\right)
$$

Then

$$
\begin{aligned}
& z_{\alpha}^{*}=\exp \left\{-\left(1-\rho+\sqrt{1+\frac{2 \rho(2+\rho)}{1+\alpha}}\right)\right\}, \\
& v_{\alpha}^{*}=\left(1+\sqrt{1+\frac{2 \rho(2+\rho)}{1+\alpha}}\right) z_{\alpha}^{*},
\end{aligned}
$$

or, equivalently,

$$
z_{\alpha}^{*}=\mathrm{e}^{-\sigma}, \quad v_{\alpha}^{*}=(\rho+\sigma) \mathrm{e}^{-\sigma},
$$

if we put

$$
\sigma=1-\rho+\sqrt{1+\frac{2 \rho(2+\rho)}{1+\alpha}}
$$

Proof. Write $\psi_{\alpha}(z)$ for $\psi(z)$. Since $f(v)$ is given by (3.25), a straightforward calculation from (4.5) leads to

$$
\begin{array}{rlr}
\psi_{\alpha}(z) & =\frac{z}{2(1-\alpha)} \int_{\max (\alpha, z)}^{1}\left\{\log ^{2}\left(\frac{v}{z}\right)+\frac{2(1-v)}{v} \log \left(\frac{v}{z}\right)\right\} \mathrm{d} v \\
& = \begin{cases}\frac{z}{1 \frac{z}{z}}\left[1-2 z+\{p(z)+1\}^{2}\right] & \text { if } \alpha<z, \\
\left.\frac{1-\alpha}{1-\alpha} \alpha+\{p(z)+1\}^{2}-\alpha\left\{q_{\alpha}(z)+1\right\}^{2}\right]-\frac{z}{2}\left\{q_{\alpha}(z)+2\right\}^{2} & \text { if } z \leq \alpha,\end{cases}
\end{array}
$$


where $p(z)$ and $q_{\alpha}(z)$ are as given in (3.27) and (3.28), respectively. Differentiating $\psi_{\alpha}(z)$ with respect to $z$ yields

$$
\psi_{\alpha}^{\prime}(z)= \begin{cases}\frac{1}{1-\alpha}\left[\{p(z)\}^{2}-4 z\right] & \text { if } \alpha<z \\ \frac{1}{1-\alpha}\left[\{p(z)\}^{2}-\alpha\left\{q_{\alpha}(z)\right\}^{2}\right]-\frac{1}{2} q_{\alpha}(z)\left(q_{\alpha}(z)+2\right) & \text { if } z \leq \alpha .\end{cases}
$$

By considering

$$
\begin{aligned}
\frac{1}{1-\alpha} & {\left[\{p(z)\}^{2}-\alpha\left\{q_{\alpha}(z)\right\}^{2}\right]-\frac{1}{2} q_{\alpha}(z)\left(q_{\alpha}(z)+2\right) } \\
& =\frac{1}{2}\left[\{p(z)\}^{2}+2(1+\rho) p(z)-\frac{1-\alpha}{1+\alpha} \rho(2+\rho)\right] \\
& =\frac{1}{2}[p(z)+2-\sigma][p(z)+2 \rho+\sigma]
\end{aligned}
$$

we can write (4.8) as

$$
\psi_{\alpha}^{\prime}(z)= \begin{cases}\frac{1}{1-\alpha}[p(z)-2 \sqrt{z}][p(z)+2 \sqrt{z}] & \text { if } \alpha<z \\ \frac{1}{2}[p(z)+2-\sigma][p(z)+2 \rho+\sigma] & \text { if } z \leq \alpha\end{cases}
$$

Observe now that, for $0<z<1$ and $0<\alpha<1$,

$$
p(z)+2 \rho+\sigma>0
$$

or, equivalently,

$$
2 \rho+\sigma-2>\log z .
$$

To prove this, it suffices to show that the left-hand side is positive. This, in fact, follows since $\rho$ in (4.6) is decreasing in $\alpha$ with $\lim _{\alpha \rightarrow 1} \rho=0$ implying that, for $0<\alpha<1, \rho>0$ and so $\sigma+\rho=1+\sqrt{1+2 \rho(2+\rho) /(1+\alpha)}>2$ from (4.7).

From (3.19) and (4.10), the sign of $\psi_{\alpha}^{\prime}(z)$ in (4.9) coincides with that of the function $\eta_{\alpha}(z)$ (continuous with respect to $z$ ) defined as

$$
\eta_{\alpha}(z)= \begin{cases}p(z)-2 \sqrt{z} & \text { if } \alpha<z \\ C(\alpha)[p(z)+2-\sigma] & \text { if } z \leq \alpha\end{cases}
$$

where $C(\alpha)$ is a positive function of $\alpha$ given as

$$
C(\alpha)=\frac{(1-\alpha)[p(\alpha)+2 \rho+\sigma]}{2[p(\alpha)+2 \sqrt{\alpha}]} .
$$

We can show that the equation $\eta_{\alpha}(z)=0$ has a unique solution whose value is greater than or equal to $\alpha$ for $\alpha \leq \alpha^{*}$, but less than $\alpha$ for $\alpha>\alpha^{*}$ by the same reasoning as used in the proof of Lemma 3.2. Taking this into consideration, we obtain $z_{\alpha}^{*}$ as a unique root $z$ of $\eta_{\alpha}(z)=0$ and then $v_{\alpha}^{*}=\psi\left(z_{\alpha}^{*}\right)$.

See Table 1 for the values of $z_{\alpha}^{*}$ and $v_{\alpha}^{*}$. Inspection of this table reveals that $z_{\alpha}^{*}$ is increasing in $\alpha\left(\geq \alpha^{*}\right)$ and larger than the corresponding values for model 1. Interestingly, $v_{\alpha}^{*}$ is not increasing in $\alpha$, but seems unimodal with its peak near $\alpha=0.2$. More elaborate calculations 
show that the peak is flat, i.e. the maximal value $v_{\alpha}^{*}=0.286316$ is the same to the 6 th decimal place for $\alpha \in[0.2009,0.2033]$. As a whole, the variation of $v_{\alpha}^{*}$ is rather small, staying between 0.253 and 0.283 .

To examine a general property of $\psi(z)$ in (4.5), differentiate $\psi(z)$ with respect to $z$. Then

$$
\psi^{\prime}(z)=\frac{1}{z} \psi(z)-\int_{z}^{1}\left\{\log \left(\frac{v}{z}\right)+\frac{1-v}{v}\right\} f(v) \mathrm{d} v .
$$

Thus, the optimal $z\left(=z^{*}\right)$ satisfies $\psi^{\prime}(z)=0$, or, equivalently,

$$
\int_{z}^{1}\left[\frac{1}{2} \log \left(\frac{v}{z}\right)\left\{\log \left(\frac{v}{z}\right)-2\right\}+\frac{1-v}{v}\left\{\log \left(\frac{v}{z}\right)-1\right\}\right] f(v) \mathrm{d} v=0,
$$

implying that $z^{*} \leq \mathrm{e}^{-1}$, because $\log (v / z)-2<\log (v / z)-1<0$ for $\mathrm{e}^{-1}<z<v<1$. This property can be seen, in a sense, as a limiting version of Lemma 2.3 , because, in the secretary case, $s^{*}(n)=s_{c}^{*}(n) \approx \mathrm{e}^{-1} n$ as $n \rightarrow \infty$ in (2.13). The optimal payoff is given by (from (4.11))

$$
v^{*}=\psi\left(z^{*}\right)=z^{*} \int_{z^{*}}^{1}\left\{\log \left(\frac{v}{z^{*}}\right)+\frac{1-v}{v}\right\} f(v) \mathrm{d} v .
$$

For the curtailed geometric prior, we have the following result. The proof is omitted since it is similar to that of Lemma 3.3.

Lemma 4.3. (Curtailed geometric prior.) We use the notation $z_{c}^{*}$ and $v_{c}^{*}$ for $z^{*}$ and $v^{*}$, respectively. Then $z_{c}^{*}$ is a unique root $z \in(0,1)$ of the equation

$$
\mathrm{e}^{-c z}-\mathrm{e}^{-c}-\mathrm{e}^{-c} \log z\left(2+\frac{1}{2} \log z\right)=(1+c) J_{c}(z)+\{1+(1+c) \log z\} I_{c}(z),
$$

where $I_{c}(z)$ and $J_{c}(z)$ are as given in Lemma 3.3. Moreover,

$$
v_{c}^{*}=\frac{z_{c}^{*}}{1-\mathrm{e}^{-c}}\left[\mathrm{e}^{-c}-\mathrm{e}^{-c z_{c}^{*}}+\mathrm{e}^{-c} \log z_{c}^{*}+(1+c) I_{c}\left(z_{c}^{*}\right)\right] .
$$

See Table 2 for the values of $z_{c}^{*}$ and $v_{c}^{*}$. These values are both decreasing in $c$. In contrast to the generalized uniform case, the threshold value $z_{c}^{*}$ of model 2 is smaller than the corresponding value of model 1 . However, $v_{c}^{*}$ varies little, staying between 0.233 and 0.253 . It is interesting to compare this duration problem with the corresponding best-choice problem considered in Tamaki (2011, Lemma 3.2 and Table 2 for $m=1$ ). As $c \rightarrow \infty$, both problems seem to have the same asymptotic optimal value of 0.2329 . This is intuitive because, depending on whether the chosen object is best overall (i.e. the last candidate) or not, the resulting duration tends to 1 or 0 from $\lim _{c \rightarrow \infty} \mathbb{E}\left[V_{c}\right]=0$.

\section{Acknowledgements}

The author is grateful to Dr. Q. Wang for her help with the numerical evaluations and to the anonymous referee for useful comments.

\section{References}

Bruss, F. T. (2000). Sum the odds to one and stop. Ann. Prob. 28, 1384-1391.

Dynkin, E. B. And Yushkevich, A. A. (1969). Markov Processes: Theorems and Problems. Plenum Press, New York. FErguson, T. S. (2008). The sum-the-odds theorem with application to a stopping game of Sakaguchi. Preprint. 
Ferguson, T. S., Hardwick, J. P. And TAMAKI, M. (1992). Maximizing the duration of owning a relatively best object. In Strategies for Sequential Search and Selection in Real Time (Contemp. Math. 125), American Mathematical Society, Providence, RI, pp. 37-57.

Gilbert, J. P. And Mosteller, F. (1966). Recognizing the maximum of a sequence. J. Amer. Statist. Assoc. 61, $35-73$. Gnedin, A. V. (2004). Best choice from the planar Poisson process. Stoch. Process. Appl. 111, 317-354.

Gnedin, A. V. (2005). Objectives in the best-choice problems. Sequent. Anal. 24, 177-188.

Mazalov, V. V. AND TAMAKI, M. (2006). An explicit formula for the optimal gain in the full-information problem of owning a relatively best object. J. Appl. Prob. 43, 87-101.

Mucci, A. G. (1973a). Differential equations and optimal choice problem. Ann. Statist. 1, 104-113.

Mucci, A. G. (1973b). On a class of secretary problems. Ann. Prob. 1, 417-427.

Pearce, C. E. M., Szajowski, K. And TAMaki, M. (2012). Duration problem with multiple exchanges. Numer. Algebra Control Optimization 2, 333-355.

Petruccelli, J. D. (1983). On the best-choice problem when the number of observations is random. J. Appl. Prob. 20, 165-171.

Presman, E. L. And Sonin, I. M. (1972). The best choice problem for a random number of objects. Theory. Prob. Appl. 17, 657-668.

Samuels, S. M. (2004). Why do these quite different best-choice problems have the same solutions? Adv. Appl. Prob. 36, 398-416.

Suchwalko, A. AND Szajowski, K. (2002). Non standard, no information secretary problems. Sci. Math. Japonicae 56, 443-456.

Tamaki, M. (2010). Sum the multiplicative odds to one and stop. J. Appl. Prob. 47, 761-777.

TAMAKI, M. (2011). Maximizing the probability of stopping on any of the last $m$ successes in independent Bernoulli trials with random horizon. Adv. Appl. Prob. 43, 760-781. 